\title{
Implication of miR-155-5p and miR-143-3p in the vascular insulin resistance and instability of human and experimental atherosclerotic plaque
}

\section{Paula González-López}

Complutense University of Madrid

\section{Carla Ares-Carral}

Complutense University of Madrid

Andrea R. López-Pastor

Complutense University of Madrid

Jorge Infante-Menéndez

Complutense University of Madrid

Tamara Gonzalez-Illanes

Complutense University of Madrid

\section{Melina Vega Céniga}

Hospital de Galdakao-Usansolo

\section{Leticia Esparza}

Hospital de Galdakao-Usansolo

\section{Nuria Beneit}

Complutense University of Madrid José Luis Martín-Ventura

IIS-Fundation Jimenez-Diaz, Autonoma University of Madrid, CIBERCV

Oscar Escribano

Complutense University of Madrid

Almudena Gómez-Hernández ( $\nabla$ algomezh@ucm.es )

Complutense University of Madrid

\section{Research Article}

Keywords: Atherosclerosis, miR-155-5p, miR-143-3p, unstable plaque, apoptosis, AKT, IGF-IIR

Posted Date: May 9th, 2022

DOI: https://doi.org/10.21203/rs.3.rs-1177585/v2 
License: (c) (i) This work is licensed under a Creative Commons Attribution 4.0 International License. Read Full License 


\section{Abstract}

Background: Cardiovascular diseases (CVDs) are the main cause of death in first world countries, being atherosclerosis, a recurring process underlying their apparition. MicroRNAs (miRNAs) are small noncoding RNAs that modulate the expression of their target proteins. Therefore, they have emerged as key players in diseases like cancer, diabetes, or CVDs.

Methods: Apolipoprotein E-deficient $\left(A p o E^{-}\right)$mice fed a standard type diet (STD) or high fat diet (HFD) for 8 and 18 weeks was compared to wild type (WT) STD-fed groups for the same time. 18 miRNAs were selected (from Pubmed and GEO database) for their possible role in promoting atherosclerosis and were analysed by RT-qPCR in the aorta from the experimental model. Afterwards, the altered miRNAs in the aorta from 18 weeks- $A p o E^{-1}$ mice were studied in human healthy aortic samples, human early aortic atherosclerotic plaques, and human advanced carotid atherosclerotic plaques.

Results: From the 18 miRNAs analyzed, miR-155-5p was overexpressed and miR-143-3p was downregulated in mouse and human atherosclerotic lesions. In addition, a significant decrease of protein kinase $B(A K T)$, target of miR-155-5p, and an increase of insulin-like growth factor type II receptor (IGFIIR), target of miR-143-3p, were noted in aortic roots from $A p o E^{-/}$mice and in carotid plaques from ACA patients. Finally, both miRNAs were studied on vascular endothelial and smooth muscle cell lines. The overexpression of miR-155-5p reduced AKT levels and its phosphorylation in vascular smooth muscle cells. MiR-143-3p overexpression decreased IGF-IIR reducing apoptosis in vascular cells.

Conclusions: Our results suggest that miR-155-5p and miR-143-3p may be implicated in insulin resistance and plaque instability by the modulation of their targets AKT and IGF-IIR, contributing to the progression of experimental and human atherosclerosis.

Trial Registration: authorization numbers PFS09-007 and PI1442016.

\section{Background}

Cardiovascular diseases are the main cause of premature death in first world countries and usually progress with an asymptomatic period [1]. Atherosclerosis is usually the underlying cause of cardiovascular diseases like coronary artery disease or myocardial infarction [2-4].

Insulin-resistant states are associated with metabolic abnormalities that include glucotoxicity, lipotoxicity and inflammation, and which also lead to endothelial dysfunction. Therefore, hyperglycaemia, hyperlipidemia and proinflammatory cytokines are known to selectively impair the phosphoinositide 3kinase (PI3K)/AKT/endothelial nitroxide synthase (eNOS) pathway, increase oxidative stress and enhance the release of endothelin 1 (ET-1) from the endothelium [5]. Different manifestations associated to insulin resistance, including dyslipidemia, hyperglycaemia, inflammation and obesity, may be intermediary mediators together to insulin resistance to generate endothelial dysfunction $[6,7]$. 
MicroRNAs (miRNA) are small non-coding RNAs conserved between species that regulate the expression of genes. Since their discovery, hundreds of these transcripts have been described unravelling the role they play in development or disease [8].

The effect of miR-155-5p in several diseases is controversial. Some studies demonstrated that this miRNA protects against the progression of diseases like cervical cancer by targeting phosphoinositidedependent protein kinase 1 (PDK1)[9] or type-2-diabetes by targeting the transcription factor MafB (Mafb) [10]. During atherosclerosis progression, miR-155-5p has been proven to be overexpressed promoting inflammation [11] and oxidized low-density lipoprotein uptake in macrophages [12].

The miR-143-3p is well studied in the progress and development of different types of cancer like adenocarcinoma [13] or ovarian cancer [14]. In recent years, miR-143-3p has emerged as a possible regulator of myocardial infarction $[15,16]$. In contrast, the role this miRNA plays in atherosclerosis remains mostly unknown. For these reasons, in the current paper we studied the role of miR-155-5p and miR-143-3p and their targets (AKT and IGF-IIR, respectively) in the progression of experimental and human atherosclerosis, as well as its implication in the vascular insulin resistance. To carry out this objective, we have developed a classic experimental model of atherosclerosis, such as the $A p o E^{-/-}$ mouse under a standard diet (STD) or a high-fat diet (HFD) for 8 and 18 weeks. To address the role in human atherosclerosis we have used vascular samples from healthy subjects, subjects with fibrolipidic plaques (FL) or with advanced carotid atherosclerosis (ACA). Finally, to delve into the effect that both miRNAs could have in the atherosclerotic process and in vascular insulin resistance, we performed in vitro experiments on human vein endothelial cells (HUVECs) and vascular smooth muscle cells (VSMCs).

\section{Methods}

\section{Human samples}

We used two cohorts of patients. In the first of them, the human samples $(n=14)$ were obtained from human infradiaphragmatic aortic segments from 7 healthy subjects and 7 subjects with fibrolipidic lesions. Each of them was harvested from a different donor after organ transplantation with the authorization of the French Biomedicine Agency (authorization number PFS09-007). There were no significant differences in terms of age and gender. The investigation conforms to the principles outlined in the Declaration of Helsinki.

The second cohort corresponds to patients with advanced carotid atherosclerosis. Forty atherosclerotic plaques from patients with carotid stenosis $>70 \%$ undergoing carotid endarterectomy at IIS-Fundación Jiménez Díaz (Supplemental Table 1). The plaques contained an important proportion of inflammatory cells (Stary stages $\mathrm{V}-\mathrm{VI}$ ), whereas adjacent areas were mainly composed of VSMCs and lipid deposits (Stary stage III). The study was approved by the hospital's ethics committee (IIS-Fundación Jiménez Díaz) with the reference number PI1442016 according to the institutional and the Good Clinical Practice 
guidelines, which was performed in accordance with the Declaration of Helsinki. All participants gave written informed consent.

\section{Animal model}

Male C57BI/6 Wild type (WT) and $A p o E$ knockout $\left(A p o E^{\prime-}\right)$ mice were maintained under standard light (12 hours long light/dark cycles), temperature $\left(23.3^{\circ} \mathrm{C}\right.$ ), and humidity $(65.1 \%)$ conditions, and ad libitum diet from their weaning, up to their sacrifice. All the procedures were handled according to the rules set by the ethical committee from the Universidad Complutense de Madrid and Autonomic Community of Madrid (PROEX188/88). All animal procedures performed must conform to the guidelines from Directive 2010/63/EU of the European Parliament on the protection of animals used for scientific purposes or the current $\mathrm{NIH}$ guidelines. The microbiological and health state of the mice was controlled by the FELASA (Federation of European Laboratory Animal Science Associations) criterium and showed no pathogenic infection. The WT mice $(n=7)$ were fed a standardtype diet ([STD] $3 \%$ of the kcal are given by fat, Emvigo, USA) for 8 or 18 weeks, while the $A p o E^{-1-}$ were separated in two groups: one was fed the STD $(n=7)$, and the other a HFD $(n=10)(60 \%$ of the kcal are given by fat, Emvigo, USA) for 8 or 18 weeks before sacrifice.

In order to study insulin signaling in physiological conditions, in vivo insulin signalling assays were performed. Fasted mice were intraperitoneally (i.p.) injected with $1 \mathrm{U} / \mathrm{kg} \mathrm{BW}$ of insulin glulisine (Apidra SoloStar, Sanofi, Paris, France) or an equivalent volume of $0,9 \% \mathrm{p} / \mathrm{v}$ saline solution ( $n=5$ per group). After 10 minutes, mice were sacrificed and harvested tissues were immediately frozen in liquid nitrogen. Insulin signalling was assessed by Western blot against phospho-AKT (Ser473) in homogenates of aorta artery.

The euthanasia was performed at 8 or 18 weeks. For it, the mice were anesthetized and by a Ketamine (50ng/mL, Ketalar ${ }^{\circledR}$ [Pfizer, USA]) and Xylazine (200mg/mL Rompun® [Bayer, Germany]) intraperitoneal injection on a $50: 5$ dose per $\mathrm{Kg}$. Then the aorta and the liver were recovered and stored at $-80^{\circ} \mathrm{C}$, while the aortic root was washed with saline and then included in Tissue-Tek® O.C.T. Compound ([O.C.T.], VWR $\mathrm{BDH}$ Chemicals $\AA$, USA) and stored at $-80^{\circ} \mathrm{C}$ for further analysis, both tissues were extracted under sterile conditions. Blood was extracted from the jugular vein and citrated with $0,4 \% \mathrm{p} / \mathrm{v}$ Citrate (Merck, USA), then the plasma was recovered after a $1200 \mathrm{Xg}$ centrifuge for 15 minutes at $4^{\circ} \mathrm{C}$ for subsequent testing. Just before carrying out this procedure, the animals were weighted, and plasma glucose was measured using the Accu-check ${ }^{\circledR}$ glucometer (Aviva, Roche, Switzerland). Finally, cholesterol and triglycerides were tested in plasma samples from fasted mice (Spinreact, Spain).

\section{Cell Culture}

HUVECs were purchased from PromoCell (C-12203, Merck, USA) and cultured with MCB131 medium (Gibco ${ }^{\mathrm{TM}}$, Fisher Scientific, USA) enriched with L-glutamine $2 \mathrm{mM}$ (Gibco ${ }^{\mathrm{TM}}$, Fisher Scientific, USA), foetal bovine serum 7,5\% v/v (Gibco ${ }^{\mathrm{TM}}$, Fisher Scientific, USA), Penicillin/Streptomycin $100 \mathrm{U} / \mathrm{mL}\left(\mathrm{Gibco}^{\mathrm{TM}}\right.$, Fisher Scientific, USA) and endothelial growth factor $1 \mathrm{X}$ (R\&D systems ${ }^{\circledR}$, USA) and maintained at $37^{\circ} \mathrm{C}$ in a humified $5 \% \mathrm{CO}_{2}$ incubator (Fisher Scientific, USA). 
Generation of immortalized WT VSMCs lines was previously described [17]. Cell lines were cultured to subconfluence (70-80\%) with 10\% foetal bovine serum (FBS)-DMEM for in vitro experiments.

\section{Histological tissue samples}

The paraffin embedded human carotids or livers from experimental model were cut into $5 \mu \mathrm{m}$ sections and stained with hematoxylin and eosin purchased from PanReac Appli Chem ITW Reagents and Masson-Goldner staining kit (1.00485.0001, Sigma-Aldrich, USA). AKT and IGF-IIR were detected by immunoperoxidase with rabbit anti-AKT (\#9272, Cell Signalling Technology Inc $\circledast$, USA) and anti-IGF-IIR (sc-25462, Santa Cruz Biotechnology, USA) polyclonal antibodies. After an overnight incubation with each primary antibody, we incubated with a peroxidase-conjugated secondary antibody for $1 \mathrm{~h}$ at 1:100 dilutions. The sections were stained for $10 \mathrm{~min}$ at room temperature with 3,3-diaminobenzidine and then counterstained with hematoxylin and mounted in DPX mounting medium (255254.1610, PanReac AppliChem ITW Reagent, Sigma-Aldrich, USA). In each experiment, negative controls without the primary antibody were included to check for nonspecific staining.

The immunohistochemistry images were quantified using the "count and measure objects" tool in the Image-Pro Plus software (IPWin4.5 software). The colour considered as positive staining for the same protein was manually selected, and the value corresponding to the sum of all stained areas was obtained. The results were expressed as the percentage of the stained area with respect to the total area analyzed in each sample.

The O.C.T. embedded aortic root or liver samples from experimental model were cut into $5 \mu \mathrm{m}$ sections and an Oil Red O (Sigma-Aldrich, USA) staining contrasted with hematoxylin was performed. Oil Red O staining was analysed using Image $\mathrm{J}$ and IPWin4.5 software and we determined lipid depot, lesion area and $\%$ of stenosis per aorta area.

\section{En face imaging of aorta}

Atherosclerotic lesions were quantified by en face analysis of the whole aorta. The Oil Red-0-stained aortas were photographed, and the atherosclerotic lesions were quantified using IP Win32 v4.5 software.

\section{miRNA extraction from the aorta, liver, vascular cell lines and paraffin-embedded carotid tissue}

The miRNA content from the aorta, the liver and the cells were extracted following the mirVanaTM miRNA isolation kit (Invitrogen ${ }^{\mathrm{TM}}$, Thermo Fisher Scientific, USA). The miRNA content from paraffin-embedded carotids was extracted using the RNeasy FFPE kit (Qiagen, Germany). All the extractions were made following the protocol handled by the manufacturers. The miRNA sample concentration was then determined using a Nanodrop ${ }^{\mathrm{TM}} 2000$ and the 2000/2000c Operating Software (Thermo Scientific, USA).

\section{Cell Transfection with miRNA mimics}


miR-155-5p and miR-143-3p mimics were purchased from Sigma-Aldrich. Approximately $5 \times 10^{4}$ cells were seeded in P60 culture plates (353002, Falcon ${ }^{\mathrm{TM}}$, Thermo Fisher Scientific, USA) and were transfected with or without 10-20nM of MISSION® miRNA mimic hsa-miR143-3p or hsa-miR-155-5p (HMI0221 or HMN0254, Sigma-Aldrich, USA). INTERFERin ${ }^{\circledR}$ siRNA transfection reagent (\#409 - 10, Polyplus transfection ${ }^{\circledR}$, France) was used as a vehicle. The effect of the transfection was studied after 72 and 96 hours at a genetic and proteomic scale, respectively, as specified in the protocol. After that, the cells were deprived in $0 \%$ FBS medium for $6 \mathrm{~h}$ with or without $100 \mathrm{nM}$ insulin (Sigma-Aldrich, USA) for 10 minutes to study the effect of miR-155-5p mimic on the insulin signalling pathway. To study the effect of the miR143-3p mimic on cell apoptosis, the cells were just deprived of FBS for 6 hours or 2 hours followed by a treatment with thapsigargin for 2 hours (100 nM, Santa Cruz Biotechnology, USA).

\section{RT-qPCR analysis}

Retrotranscription of miRNA was performed using the TaqMan® Advanced miRNA cDNA Synthesis Kit (Thermo Scientific, USA) from human and murine samples or cell lines. Quantitative polymerase chain reaction (qPCR) was done using cDNA as template and the TaqMan® Fast Advanced Master Mix (Thermo Scientific, USA). The genes were detected using TaqMan ${ }^{\circledR}$ (Thermo Scientific, USA) primers for hs-miR-143-3p (477912_mir, mature miRNA sequence: UGAGAUGAAGCACUGUAGCUC), mmu-miR-155-5p (mmu480953_mir, mature miRNA sequence: UUAAUGCUAAUUGUGAUAGGGGU) and mmu-miR-191-5p (mmu481584_mir, mature miRNA sequence: CAACGGAAUCCCAAAAGCAGCUG) used as endogenous gene. All the primers detect both mouse and human target gene.

\section{Western blot analysis}

Proteins from cell lysates $(20-60 \mu \mathrm{g})$, and tissue samples $(20-70 \mu \mathrm{g})$ were separated on a $10 \%$ or $10-$ $20 \%$ gradient $30 \%$-acrylamide gel and then transferred to a $0.45 \mu \mathrm{M}$ pore PVDF membrane (Merck, USA) as we previously described[17]. The antibodies used are described in the Supplemental Table 2. The bands were visualized using the Clarity Western Blot Analysis ECL (BioRad ${ }^{\circledR}$, USA). The protein bands density was analysed with ImageJ Software 1.52a (Java 1.8.0_112).

\section{Database search to find miRNAs and their possible targets}

A thorough search in GEO database, Target Scan, miRWalk, miRDB, DIANA and miRTarBase was used to search for miRNAs and their possible targets.

\section{Statistical analysis}

The data from the experimental groups was analysed using GraphPad Prism v8. A Normality and Lognormality test was done to confirm that the data had a normal distribution, afterwards a T-Student or ANOVA test were carried out if there were two or more groups, respectively. Correlation between variables was assessed by two-tailed Spearman's $r$ correlation analyses. All the graphs were designed using the same software.

\section{Results}




\section{miR-143-3p and miR-155-5p levels are altered in aorta from ApoE $^{-/-}$mice}

We used a classic model of atherosclerosis in mice as $\mathrm{ApOE}^{-/-}$model under HFD. Firstly, we confirmed that $\mathrm{ApOE}^{-/-}$mice had a significant increase of body weight and weight gain in addition to hypercholesterolemia and hypertriglyceridemia (Supplemental Table 3), being significantly higher in $A p o E$ ${ }^{-/-}$fed with HFD for 18 wks.

After that, we analysed vascular damage and performed an Oil Red O (ORO) staining contrasted with hematoxylin in aortic roots (Fig. 1A). A significant increase of lipid depot, lesion area and \% of stenosis in aortic roots from $\mathrm{ApoE}^{/-}$HFD 18wks were noted in comparison with the other groups (Fig. 1B). Similarly, en face analysis of ORO-stained whole aorta showed a significant increase of lesion area in $\mathrm{ApoE}^{-1-}$ under HFD for 18 weeks vs. Control STD and $A p o E^{-/-}$STD of the same age (Supplementary Fig. 1).

PubMed and GEO Databases were used to perform a search of miRNAs that could play a role in atherosclerosis. After the search, a screening of the selected miRNAs was performed in the aorta of the different 8- and 18-weeks mice fed with STD and HFD (Supplemental Table 4). From the selected miRNAs: miR-155-5p was significantly overexpressed in $A p E^{-1}$ mice after 8 weeks of HFD (Fig. 1C, left) and after 18 weeks of HFD compared with each WT mice (Fig. 1C, right); and miR-143-3p was significantly downregulated in $A p o E^{-/}$mice after 18 weeks of HFD vs. WT mice (Fig. 1D, right).

\section{miR-143-3p and miR-155-5p levels are also altered in human atherosclerotic carotid plaque}

To confirm whether the levels of the studied miRNAs in the murine model of atherosclerosis might have a clinical relevance in human atherosclerosis, we analysed the levels of different miRNAs in two cohorts: healthy subjects $(\mathrm{H})$, subjects with fibrolipidic plaque $(\mathrm{FL})$ and patients with human advanced atherosclerosis undergoing carotid endarterectomy (ACA) (Supplemental Table 4). Firstly, we performed a Masson staining, and we could differentiate regions as media in samples from $\mathrm{H}$ and $\mathrm{FL}$ and media, fibrous and shoulders in samples from ACA (Fig. 2A). Histological analysis revealed that complicated plaques from ACA contained an intraplaque haemorrhage and/or a certain degree of calcification with a relatively important proportion of inflammatory cells. The adjacent non-complicated regions were composed of fibrous thickening with a variable content of VSMCs (Fig. 2A).

In serial sections of samples used to histological characterization, we isolated miRNAs and analysed the levels of miR-155-5p (Fig. 2B) and miR-143-3p (Fig. 2C) by qPCR, showing that miR-155-5p expression was significantly upregulated (Fig. 2B) and miR-143-3p downregulated (Fig. 2C) in the ACA patients compared with healthy subjects and subjects with fibrolipidic plaques.
AKT expression is modulated by miR-155-5p levels in atherosclerosis and non-alcoholic fatty liver disease 
To study the effect of miR-155-5p overexpression and its implication in atherosclerosis and vascular insulin resistance, HUVECs and VSMCs, were transfected with pre-miRNA 155-5p, a mimic specific for miR-155-5p. After 72h of transfection, miR-155-5p was overexpressed in both vascular cells (Fig. 3A). Following an extensive search in different databases, AKT, eNOS and p85a stood out as three possible miR-155-5p targets (Supplemental Fig. 2A and B).

After $96 \mathrm{~h}$ of transfection with the miR-155-5p mimic, eNOS and p85a were significantly downregulated in HUVECs (Fig. 3B and C) and VSMCs (Fig. 3D), respectively. AKT expression was also significantly downregulated in HUVECs (Fig. 3B and C) and VSMCs (Fig. 3D). Therefore, AKT expression was also significantly downregulated in the aorta of the 18 weeks-fed $A p o E^{-/-}$mice (Fig. $4 \mathrm{~A}$ ) and in the carotid from patients with advanced carotid atherosclerosis (Fig. 4B). We also established a negative correlation between miR-155-5p and its target, AKT, in the carotid of patients with atherosclerosis (Fig. 4C).

In addition to AKT levels was decreased by miR-155-5p overexpression, we wanted to confirm whether its activation was also affected. In VSMCs, we observed that cells transfected with pre-miR-155-5p showed a lower AKT phosphorylation induced by $100 \mathrm{nM}$ insulin for $10 \mathrm{~min}$ (Fig. 3E). This fact was confirmed in the experimental model by in vivo insulin signalling study (Fig. 4D). In aorta from ApoE ${ }^{-/-}$HFD 18 wks, with high levels of miR-155-5p, insulin administration did not induce AKT phosphorylation. However, insulin induced a significant increase of AKT phosphorylation in aorta from WT STD 18wks and ApoE ${ }^{-1-}$ STD 18wks (Fig. 4D).

To confirm if the levels of the miR-155-5p might be altered in function of grade of obesity. The patients were separated by their body mass index (BMI) following the criteria established by the World Health Organization (lean, overweight and obese) and we analysed miR-155-5p and AKT. So, miR-155-5p showed a significant increase expression-wise (Fig. 5A), and a progressive decrease of AKT levels (Fig. 5B) in accordance with BMI, which was confirmed via Spearman's correlation (Fig. 5C and D). In addition, we also separated the patients in diabetic and non-diabetic, obtaining a high and significant increase of miR155-5p in diabetic patients with ACA (Supplemental Fig. 3).

Since the 18 weeks-fed $\mathrm{ApoE}^{-/-}$mice model also showed hepatic steatosis (Fig. 6A), the expression of miR-155-5p was analysed in the liver by qPCR. So, an increase of miR-155-5p was noted in liver from $A p o E^{/-}$mice (Fig. 6B), which correlated with a downregulation in AKT expression in liver from $A p o E^{-1-}$ mice (Fig. 6C).

\section{IGF-IIR expression is modulated by miR-143-3p}

To study the effect of miR-143-3p downregulation and its role in the mechanisms involved in atherosclerosis progression, HUVECs and VSMCs were transfected with a specific miRNA mimic to increase miR-143-3p expression. After $72 \mathrm{~h}$ of transfection, miR-143-3p expression was significantly increased in both HUVECs (Fig. 7A) and VSMCs (Fig. 7B). After search in different databases, IGF-IIR stood out as a possible target (Supplemental Fig. 2C and D). 
Succeeding $96 \mathrm{~h}$ of transfection with the miRNA mimic, IGF-IIR expression was significantly downregulated in HUVECs (Fig. 7C), and in VSMCs (Fig. 7D). In the same way, in the aorta of 18 wks-HFD$A p o E^{-1}$ mice, miR-143-3p expression was downregulated and IGF-IIR expression was increased (Fig. 1C and 7C, respectively). Moreover, miR-143-3p was downregulated and IGF-IIR was significantly overexpressed in the shoulder regions in carotid plaque from ACA patients (Fig. 2C and 7D).

Since IGF-IIR has been implicated in the instability of advanced atherosclerotic plaques, we performed new experiments to elucidate the relationship between miR-143-3p, IGF-IIR and apoptosis. For that, we induced the activation of caspase 3 by FBS starvation for 6 hours in HUVECs or 100 nM thapsigargin treatment for 2 hours in VSMCs (Fig. 7E). More importantly, when HUVECs and VSMCs were pre-treated with pre-miR-143-3p for $96 \mathrm{~h}$ the levels of active caspase 3 was significantly reduced in basal conditions or in HUVECs with FBS starvation for 6h (HUVECs) or in VSMCs stimulated with thapsigargin (Fig. 7E).

\section{Discussion}

Atherosclerosis is a silent and asymptomatic disease for decades in which various factors can contribute to the progression and rupture of vulnerable plaques and, consequently, trigger the acute event such as acute myocardial infarction or stroke [18]. The study of new biomarkers as miRNAs might help to identify the presence of vulnerable plaques and to be able to avoid the progression process. In this study, we have studied 18 miRNAs and finally, have identified two miRNAs, miR-155-5p and miR-143-3p, with altered levels in both, an experimental atherosclerosis model in mice and in patients with carotid advanced atherosclerosis. In this paper, we presented a novel role of miR-155-5p and miR-143-3p in the insulin resistance and apoptosis of vascular resident cells, respectively, being both key processes in the progression and instability of atherosclerotic plaque.

miR-155 is one of the most dynamically regulated and multifunctional miRNAs, which has been associated with the regulation of immune-related processes, with an impact on cancer $[8,9]$ and atherosclerosis [19]. Diverse studies about the role of miR-155-5p in atherosclerosis have obtained controversial results and the plausible explanation might be that the function of miR-155-5p depend on the phase of atherosclerosis. The miR-155-5p suppresses atherosclerosis in the early phase, while it shows the opposite effect in the advanced phase [20]. So, in a model of early atherosclerosis, low-density lipoprotein receptor deficient mice transplanted with miR-155-deficient bone marrow had increased atherosclerotic plaques, elevated levels of pro-inflammatory monocytes, and decreased interleukin (IL)-10 production [21]. However, several studies using $A p O E^{-/-}$mice as a model for advanced phase of atherosclerosis demonstrated that the injection of antagomir-155 attenuated atherosclerosis development and progression in $\mathrm{ApoE}^{-/}$mice [22] or the plaque size was decreased in $\mathrm{ApoE}^{-/}$mice with a leukocyte specific miR-155 deficiency [23] or in $A p o E^{/-}$miR-155 ${ }^{-/-}$double knockout mice [24]. According to these results, we obtained a significant increase of miR-155-5p in aortic roots from $A p o E^{1-}$ fed with HFD, a model of advanced atherosclerosis, as well as in carotid biopsies from patients with advanced atherosclerosis. In contrast, subjects with fibrolipidic plaques, an early phase of 
atherosclerosis, showed lower miR-155-5p levels than healthy subjects. In this regard, previous studies have described that miR-155-5p might be a possible biomarker of plaque instability in the plasma of coronary artery disease (CAD) patients [25] and overexpressed in macrophages during atherosclerosis progression [26]. miR-155 has also been described to promote inflammatory activation of macrophages by repressing B-cell leukemia/lymphoma (BCL-6), a negative regulator of nuclear factor-kappa B (NF-KB) signaling, thus promoting atherosclerosis [25].

The following step was to identify a target of miR-155-5p involved in atherosclerosis process and vascular insulin resistance in diverse databases and so, confirm its downregulation in vivo and in vitro. In this sense, AKT had been confirmed as a target from miR-155-5p [27]. During the atherosclerotic plaque progression, AKT promotes the proliferation and survival of endothelial cells and VSMCs $[28,29]$. We demonstrate that AKT was decreased in the aorta from $A p o E^{-/-}$mice HFD and in carotid from ACA patients, result that correlates with an overexpression of miR1555p. However, patients with early atherosclerosis presented a significant increase of AKT protein levels. We also established a significant inverse correlation between miR-155-5p and AKT in human carotid artery. Moreover, in VSMCs and HUVECs, a significant decrease of AKT protein levels was noted after miR-155-5p overexpression and in consequence, a strong decrease in AKT phosphorylation and function. Moreover, miR-155-5p also regulates p85a as we observed in VSMCs, and also might contribute to the significantly decrease of AKT phosphorylation.

Afterwards, we have found a significant and robust correlation between BMI and miR-155-5p as well as $\mathrm{BMI}$ and AKT. Recently, several miRNAs have emerged as involved in pathways related to obesity such as adipokine expression, glucose and lipid metabolism, insulin signaling, oxidative stress, and inflammation $[30,31]$. Both the adipose tissue and circulating miRNAs are deregulated in human obesity [32]. Previous studies have described that miR-155-5p together to miR-34a and let7c might have altered its expression and be modulated by tumor necrosis factor- $a$ (TNF- $a$ ) in human adipocytes and is closely associated with NF-KB signalling $[33,34]$. It has been reported that NF-KB-p65 interacts with the promoter site of miR-155, so that NF-KB activation increases levels of miR-155 [35]. In this regard, one of mechanism implicated in an increase of miR-155-5p observed in advanced atherosclerosis could be NF-KB activation itself since some groups have described a significant NF-KB activation in carotid advanced atherosclerosis [36, 37] as well as in aortic root from $A p o E^{-/-}$mice under HFD [38], being both similar to those used by us.

In this regard, modulation of this inflammation-related miR-155-5p in both adipocytes and their exosomes would improve adipocyte dysfunction and would have an impact on distant organs, such as liver or vascular tissues [39]. Concordantly with this treatment, the deletion of miR-155 in mice prevented dietinduced obesity, improved insulin sensitivity, and abrogated adipocyte hypertrophy and adipose tissue inflammation [40]. So, the reduction of miR-155 might increase expression of genes involved in brown adipogenesis, lipolysis, and energy release, which could synergize to improve fat metabolism [41-43]. We also observed that this miRNA might be implicated in diverse processes that they can occur simultaneously and coordinate in the same subject or animal, such as advanced atherosclerosis as well as the vascular and hepatic insulin resistance. So, in $A p o E^{-/-}$mice under HFD that develop advanced 
atherosclerosis and fatty liver, a significant increase of miR-155-5p levels not only was observed in aorta but also in liver. Accordingly, with our results, hepatic miR-155-5p is upregulated in HFD-induced nonalcoholic fatty liver disease (NAFLD) in rats [44] and in non-alcoholic steatohepatitis (NASH) mouse models [45]. Indeed, miR-155 plays a key role in the liver lipid metabolism and its deficiency reduces steatosis and fibrosis [46]. Therefore, miR-155-5p affects various pathways in parallel that may elicit adiposity and obesity and vascular and hepatic alterations.

The other miRNA candidate that we have studied has been miR-143-3p, which modulates the expression of several genes relevant to cardiovascular biology and function. This miRNA plays a protective role in myocardial infarction by targeting ciclooxygenase-2 [16] or apoptosis-related genes [47]. In all cases, the action of miR-143-3p might be inducing cell migration and inhibiting apoptosis. In this regard, miR-143-3p has a key role in VSMCs differentiation through its target, Ets-like gene 1, a transcriptional coactivator that is crucial for regulation of the VSMC phenotype [48]. In our model of advanced atherosclerosis and in patients with ACA, we observed a significant decrease of miR-143-3p whereas in $A p o E^{-/-}$mice after only 8 weeks of HFD and in subjects with fibrolipidic plaques, both with early atherosclerosis, miR-143-3p levels were similar or higher than their respective controls.

The following step was to determine a target of miR-143-3p, such as IGF-IIR, involved in advanced atherosclerosis. Xihua $L$ et al. confirmed IGF-IIR as a target for miR-143-3p, giving to this miRNA a protective role in patients with metabolic syndrome [49]. IGF-IIR is a foetal promoter of cell growth, survival and differentiation [50]. During atherosclerosis IGF-IIR overexpression may have a protective role in macrophages and a detrimental role in VSMCs or unstable atherosclerotic plaques [51, 52]. Moreover, IGF-IIR activates caspase 3 in cardiomyocytes, promoting apoptosis [53]. In our work, we related an increase in IGF-IIR expression with a decrease in miR-143-3p, in human advanced atherosclerotic plaques and in aortic roots from $A p o E^{-1}$ mice HFD, giving to miR-143-3p a pro-apoptotic role in HUVECs and VSMCs. However, more work needs to be done to determine the mechanism by which IGF-IIR activates caspase 3 in atherosclerosis.

\section{Conclusions}

In summary, this study demonstrates a novel role for miR-155-5p and miR-143-3p in atherosclerosis progression. miR-155-5p overexpression may be involved in vascular insulin resistance by targeting AKT, and miR-143-3p downregulation could be a pro-apoptotic mechanism by increasing IGF-IIR during plaque progression (Fig. 8).

\section{Abbreviations}

CVDs cardiovascular diseases

miRNA microRNA

ApoE $^{-/-}$ApoE deficient 
18wks 18 weeks

STD standard-type diet

HFD high-fat diet

WT wild-type

IGF-IIR insulin-like growth factor type II receptor

AKT protein kinase $B$

p85a phosphoinositide-3-kinase regulatory subunit 1

PI3K phosphoinositide 3-kinase

eNOS endothelial nitric oxide synthase

ET-1 endothelin 1

PDK1 phosphoinositide-dependent protein kinase 1

Mafb transcription factor MafB

$\mathrm{H}$ healthy subjects

FL fibrolipidic plaque

ACA advanced carotid atherosclerosis

HUVECs human umbilical vascular endothelial cells

VSMCs vascular smooth muscle cells

O.C.T. Tissue-Tek® O.C.T. Compound

ORO Oil-red O staining

IL-10 interleukin-10

CAD coronary artery disease

BCL-6 B-cell lymphoma 6 protein homolog

TNF-a tumor necrosis factor-a

BMI body mass index 
NF-kB nuclear factor-kappa B

NAFLD non-alcoholic fatty liver disease

NASH non-alcoholic steatohepatitis

\section{Declarations}

\section{Ethics approval and consent to participate}

Each of subjects (healthy without vascular lesion or with fibrolipidic plaques) was harvested from a different donor after organ transplantation with the authorization of the French Biomedicine Agency (authorization number PFS09-007). Samples from patients with advanced carotid atherosclerosis were collected and approved by the hospital's ethics committee (IIS-Fundación Jiménez Díaz) with the reference number PI1442016 according to the institutional and the Good Clinical Practice guidelines, which was performed in accordance with the Declaration of Helsinki. All participants gave written informed consent.

All the procedures of mouse model were handled according to the rules set by the ethical committee from the Universidad Complutense de Madrid (PROEX188/88).

\section{Consent for publication}

Not applicable.

\section{Availability of data and materials}

The datasets used and/or analyzed during the current study are available from the corresponding authors (algomezh@ucm.es or oescriba@ucm.es) on reasonable request.

\section{Competing interests}

The authors declare no competing or financial interests.

\section{Funding}

This work was supported by grants RTI-2018-095098-B100 from Ministerio de Ciencia e Innovación y Universidades and Santander-UCM PR75/18-21572 given to A. Gómez-Hernández and Ó. Escribano. Jorge Infante-Menéndez and Paula González-López were funded by Programa Operativo de Empleo Juvenil from Comunidad de Madrid.

\section{Authors Contributions Statement}

P. G-L: Conceptualization; Investigation; Methodology; Data curation; Writing: original draft, review \& editing. C. A-C: Conceptualization; Investigation; Methodology; Data curation. AR. L-P, J. I-M and T. G-I: 
Investigation; Methodology; Data curation. M. V-C and L. E: Conceptualization; Investigation; Resources. N. Beneit: Investigation; Methodology; Data curation. J.L. M-V: Investigation; Methodology; Writing: review \& editing. O. E and A. G-H: Conceptualization; Data curation; Formal analysis; Funding acquisition; Investigation; Methodology; Project administration; Supervision; Writing: review \& editing.

\section{Acknowledgements}

We want to thank Dr. Jean-Baptiste Michel for the transfer of human infradiaphragmatic aortic segments from healthy subjects and subjects with fibrolipidic lesions. Each of them was harvested from a different donor after organ transplantation with the authorization of the French Biomedicine Agency.

\section{References}

1. Francula-Zaninovic, S., Nola, I.A., 2018. Management of Measurable Variable Cardiovascular Disease' Risk Factors. Current Cardiology Reviews 14(3): 153-63, Doi: $10.2174 / 1573403 \times 14666180222102312$.

2. Saigusa, R., Winkels, H., Ley, K., 2020. T cell subsets and functions in atherosclerosis. Nature Reviews Cardiology 17(7): 387-401, Doi: 10.1038/s41569-020-0352-5.

3. Wolf, D., Ley, K., 2019. Immunity and inflammation in atherosclerosis. Herz 44(2): 107-20, Doi: 10.1007/s00059-019-4790-y.

4. Poller, W.C., Nahrendorf, M., Swirski, F.K., 2020. Hematopoiesis and cardiovascular disease. Circulation Research: 1061-85, Doi: 10.1161/CIRCRESAHA.120.315895.

5. Muniyappa, R., Montagnani, M., Koh, K.K., Quon, M.J., 2007. Cardiovascular Actions of Insulin. Endocrine Reviews 28(5): 463-91, Doi: 10.1210/er.2007-0006.

6. Muris, D.M.J., Houben, A.J.H.M., Schram, M.T., Stehouwer, C.D.A., 2012. Microvascular dysfunction is associated with a higher incidence of type 2 diabetes mellitus: A systematic review and metaanalysis.Arteriosclerosis, Thrombosis, and Vascular Biology 32(12): 3082-94, Doi: 10.1161/ATVBAHA.112.300291.

7. Gómez-Hernández, A., de las Heras, N., López-Pastor, A.R., García-Gómez, G., Infante-Menéndez, J., González-López, P., et al., 2021.Severe hepatic insulin resistance induces vascular dysfunction: Improvement by liver-specific insulin receptor isoform a gene therapy in a murine diabetic model. Cells 10(8): 1-14, Doi: 10.3390/cells10082035.

8. Bartel, D.P., 2018. Metazoan MicroRNAs. Cell 173(1): 20-51, Doi: 10.1016/j.cell.2018.03.006.

9. Wang, F., Shan, S., Huo, Y., Xie, Z., Fang, Y., Qi, Z., et al., 2018. MiR-155-5p inhibits PDK1 and promotes autophagy via the mTOR pathway in cervical cancer. International Journal of Biochemistry and Cell Biology 99(January): 91-9, Doi: 10.1016/j.biocel.2018.04.005.

10. Zhu, M., Wei, Y., Geißler, C., Abschlag, K., Campos, J.C., Hristov, M., et al., 2017. Hyperlipidemiainduced MicroRNA-155-5p improves $\beta$-cell function by targeting Mafb. Diabetes 66(12): 3072-84, Doi: $10.2337 / \mathrm{db} 17-0313$. 
11. Jiang, K., Hu, J., Luo, G., Song, D., Zhang, P., Zhu, J., et al., 2020. MiR-155-5p Promotes Oxalate- A nd Calcium-Induced Kidney Oxidative Stress Injury by Suppressing MGP Expression. Oxidative Medicine and Cellular Longevity 2020, Doi: 10.1155/2020/5863617.

12. Wang, G., Chen, J.J., Deng, W.Y., Ren, K., Yin, S.H., Yu, X.H., 2021. CTRP12 ameliorates atherosclerosis by promoting cholesterol efflux and inhibiting inflammatory response via the miR-1555p/LXRapathway. Cell Death and Disease 12(3), Doi: 10.1038/s41419-021-03544-8.

13. Xie, F., Li, C., Zhang, X., Peng, W., Wen, T., 2019. MiR-143-3p suppresses tumorigenesis in pancreatic ductal adenocarcinoma by targeting KRAS. Biomedicine and Pharmacotherapy 119(37): 109424, Doi: 10.1016/j.biopha.2019.109424.

14. Shi, H., Shen, H., Xu, J., Zhao, S., Yao, S.Z., Jiang, N., 2018. MiR-143-3p suppresses the progression of ovarian cancer. American Journal of Translational Research 10(3): 866-74.

15. Ma, W. ya., Song, R. jie., Xu, B. bin., Xu, Y., Wang, X. xiu., Sun, H. yue., et al., 2021.Melatonin promotes cardiomyocyte proliferation and heart repair in mice with myocardial infarction via miR-1433p/Yap/Ctnnd1 signaling pathway. Acta Pharmacologica Sinica 42(6): 921-31, Doi: 10.1038/s41401-020-0495-2.

16. Liu, K., Zhao, D., Wang, D., 2020. LINC00528 regulates myocardial infarction by targeting the miR143-3p/COX-2 axis.Bioengineered 11(1): 11-8, Doi: 10.1080/21655979.2019.1704535.

17. Gómez-Hernández, A., Escribano, Ó., Perdomo, L., Otero, Y.F., García-Gómez, G., Fernández, S., et al., 2013.Implication of insulin receptor $A$ isoform and IRA/IGF-IR hybrid receptors in the aortic vascular smooth muscle cell proliferation: Role of TNF-aand IGF-II. Endocrinology 154(7): 2352-64, Doi: 10.1210/en.2012-2161.

18. Stefanadis, C., Antoniou, C.K., Tsiachris, D., Pietri, P., 2017. Coronary atherosclerotic vulnerable plaque: Current perspectives. Journal of the American Heart Association 6(3): 1-18, Doi: 10.1161/JAHA.117.005543.

19. Zhu, J., Chen, T., Yang, L., Li, Z., Wong, M.M., Zheng, X., et al., 2012. Regulation of MicroRNA-155 in Atherosclerotic Inflammatory Responses by Targeting MAP3K10. PLoS ONE 7(11), Doi: 10.1371/journal.pone.0046551.

20. Bruen, R., Fitzsimons, S., Belton, O., 2019. MiR-155 in the resolution of atherosclerosis. Frontiers in Pharmacology 10(MAY), Doi: 10.3389/fphar.2019.00463.

21. Donners, M.M.P.C., Wolfs, I.M.J., Stöger, L.J., van der Vorst, E.P.C., Pöttgens, C.C.H., Heymans, S., et al., 2012. Hematopoietic miR155 deficiency enhances atherosclerosis and decreases plaque stability in hyperlipidemic mice. PloS One 7(4): 4-12, Doi: 10.1371/journal.pone.0035877.

22. Zhu, G.F., Yang, L.X., Guo, R.W., Liu, H., Shi, Y.K., Wang, H., et al., 2013. MiR-155 inhibits oxidized lowdensity lipoprotein-induced apoptosis of RAW264.7 cells. Molecular and Cellular Biochemistry 382(1-2): 253-61, Doi: 10.1007/s11010-013-1741-4.

23. Nazari-Jahantigh, M., Wei, Y., Noels, H., Akhtar, S., Zhou, Z., Koenen, R.R., et al., 2012. MicroRNA-155 promotes atherosclerosis by repressing Bcl6 in macrophages. Journal of Clinical Investigation 122(11): 4190-202, Doi: 10.1172/JCl61716. 
24. Du, F., Yu, F., Wang, Y., Hui, Y., Carnevale, K., Fu, M., et al., 2014. MicroRNA-155 Deficiency Results in Decreased Macrophage Inflammation and Attenuated Atherogenesis in Apolipoprotein E-Deficient Mice. Arteriosclerosis, Thrombosis, and Vascular Biology 34(4): 759-67, Doi:

10.1161/ATVBAHA.113.302701.

25. Singh, S., de Ronde, M.W.J., Kok, M.G.M., Beijk, M.A., De Winter, R.J., van der Wal, A.C., et al., 2020. MiR-223-3p and miR-122-5p as circulating biomarkers for plaque instability. Open Heart 7(1): e001223, Doi: 10.1136/openhrt-2019-001223.

26. Fitzsimons, S., Oggero, S., Bruen, R., McCarthy, C., Strowitzki, M.J., Mahon, N.G., et al., 2020. microRNA-155 Is Decreased During Atherosclerosis Regression and Is Increased in Urinary Extracellular Vesicles During Atherosclerosis Progression. Frontiers in Immunology 11(December): 1-18, Doi: 10.3389/fimmu.2020.576516.

27. Chen, L., Zheng, S.Y., Yang, C.Q., Ma, B.M., Jiang, D., 2019. MiR-155-5p inhibits the proliferation and migration of VSMCs and HUVECs in atherosclerosis by targeting AKT1. European Review for Medical and Pharmacological Sciences 23(5): 2223-33, Doi: 10.26355/eurrev_201903_17270.

28. Zegeye, M.M., Lindkvist, M., Fälker, K., Kumawat, A.K., Paramel, G., Grenegård, M., et al., 2018. Activation of the JAK/STAT3 and PI3K/AKT pathways are crucial for IL-6 trans-signaling-mediated pro-inflammatory response in human vascular endothelial cells. Cell Communication and Signaling 16(1): 1-10, Doi: 10.1186/s12964-018-0268-4.

29. Yao, X., Yan, C., Zhang, L., Li, Y., Wan, Q., 2018. LncRNA ENST00113 promotes proliferation, survival, and migration by activating $\mathrm{PI} 3 \mathrm{~K} / \mathrm{Akt} / \mathrm{mTOR}$ signaling pathway in atherosclerosis. Medicine (United States) 97(16), Doi: 10.1097/MD.0000000000010473.

30. Arner, P., Kulyté, A., 2015. MicroRNA regulatory networks in human adipose tissue and obesity.Nature Reviews Endocrinology 11(5): 276-88, Doi: 10.1038/nrendo.2015.25.

31. Ortega, F.J., Moreno, M., Mercader, J.M., Moreno-Navarrete, J.M., Fuentes-Batllevell, N., Sabater, M., et al., 2015.Inflammation triggers specific microRNA profiles in human adipocytes and macrophages and in their supernatants.Clinical Epigenetics 7(1): 1-10, Doi: 10.1186/s13148-015-0083-3.

32. Ortega, F.J., Moreno-Navarrete, J.M., Pardo, G., Sabater, M., Hummel, M., Ferrer, A., et al., 2010.MiRNA expression profile of human subcutaneous adipose and during adipocyte differentiation. PLoS ONE 5(2), Doi: 10.1371/journal.pone.0009022.

33. Ma, X., Becker Buscaglia, L.E., Barker, J.R., Li, Y., 2011. MicroRNAs in NF-kB signaling. Journal of Molecular Cell Biology 3(3): 159-66, Doi: 10.1093/jmcb/mjr007.

34. Carpi, S., Scoditti, E., Massaro, M., Polini, B., Manera, C., Digiacomo, M., et al., 2019.The Extra-Virgin Olive Oil Polyphenols Oleocanthal and Oleacein Counteract Inflammation-Related Gene and miRNA Expression in Adipocytes by Attenuating NF-KB Activation. Nutrients 11(12): 2855, Doi: 10.3390/nu11122855.

35. Subedi, A., Park, P.H., 2013. Autocrine and paracrine modulation of microRNA-155 expression by globular adiponectin in RAW 264.7 macrophages: Involvement of MAPK/NF-KB pathway.Cytokine 64(3): 638-41, Doi: 10.1016/j.cyto.2013.09.011. 
36. Martín-Ventura, J.L., Blanco-Colio, L.M., Muñoz-García, B., Gómez-Hernández, A., Arribas, A., Ortega, L., et al., 2004.NF-KB Activation and Fas Ligand Overexpression in Blood and Plaques of Patients with Carotid Atherosclerosis: Potential Implication in Plaque Instability.Stroke 35(2): 458-63, Doi: 10.1161/01.STR.0000114876.51656.7A.

37. Gómez-Hernández, A., Martín-Ventura, J.L., Sánchez-Galán, E., Vidal, C., Ortego, M., Blanco-Colio, L.M., et al., 2006.0verexpression of COX-2, Prostaglandin E Synthase-1 and Prostaglandin E Receptors in blood mononuclear cells and plaque of patients with carotid atherosclerosis: Regulation by nuclear factor-KB.Atherosclerosis 187(1): 139-49, Doi: 10.1016/j.atherosclerosis.2005.08.035.

38. López-Franco, O., Hernández-Vargas, P., Ortiz-Muñoz, G., Sanjuán, G., Suzuki, Y., Ortega, L., et al., 2006.Parthenolide modulates the NF-KB-mediated inflammatory responses in experimental atherosclerosis. Arteriosclerosis, Thrombosis, and Vascular Biology 26(8): 1864-70, Doi: 10.1161/01.ATV.0000229659.94020.53.

39. Scoditti, E., Carpi, S., Massaro, M., Pellegrino, M., Polini, B., Carluccio, M.A., et al., 2019. Hydroxytyrosol modulates adipocyte gene and mirna expression under inflammatory condition. Nutrients 11(10): 1-29, Doi: 10.3390/nu11102493.

40. Gaudet, A.D., Fonken, L.K., Gushchina, L. V., Aubrecht, T.G., Maurya, S.K., Periasamy, M., et al., 2016. MIR-155 deletion in female mice prevents diet-induced obesity. Scientific Reports 6(February): 1-13, Doi: $10.1038 /$ srep22862.

41. Kajimura, S., Seale, P., Kubota, K., Lunsford, E., Frangioni, J. V., Gygi, S.P., et al., 2009. Initiation of myoblast to brown fat switch by a PRDM16-C/EBP-ßtranscriptional complex.Nature 460(7259): 1154-8, Doi: $10.1038 /$ nature08262.

42. Huijsman, E., Van De Par, C., Economou, C., Van Der Poel, C., Lynch, G.S., Schoiswohl, G., et al., 2009.Adipose triacylglycerol lipase deletion alters whole body energy metabolism and impairs exercise performance in mice. American Journal of Physiology - Endocrinology and Metabolism 297(2): 505-13, Doi: 10.1152/ajpendo.00190.2009.

43. Li, Y., Fromme, T., Schweizer, S., Schöttl, T., Klingenspor, M., 2014. Taking control over intracellular fatty acid levels is essential for the analysis of thermogenic function in cultured primary brown and brite/beige adipocytes. EMBO Reports 15(10): 1069-76, Doi: 10.15252/embr.201438775.

44. Pan, M., Deng, Y., Zheng, C., Nie, H., Tang, K., Zhang, Y., et al., 2019. Chinese Herbal Medicine Formula Shenling Baizhu San Ameliorates High-Fat Diet-Induced NAFLD in Rats by Modulating Hepatic MicroRNA Expression Profiles 2019, Doi: 10.1155/2019/8479680.

45. Miller, A.M., Gilchrist, D.S., Nijjar, J., Araldi, E., Ramirez, C.M., Lavery, C.A., et al., 2013. MiR-155 Has a Protective Role in the Development of Non-Alcoholic Hepatosteatosis in Mice 8(8), Doi: 10.1371/journal.pone.0072324.

46. Csak, T., Bala, S., Lippai, D., Kodys, K., Catalano, D., 2015.MicroRNA-155 Deficiency Attenuates Liver Steatosis and Fibrosis without Reducing Inflammation in a Mouse Model of Steatohepatitis 3: 1-21, Doi: 10.1371/journal.pone.0129251. 
47. Liu, Q., Du, G.Q., Zhu, Z.T., Zhang, C., Sun, X.W., Liu, J.J., et al., 2015. Identification of apoptosis related microRNAs and their target genes in myocardial infarction post - transplantation with skeletal myoblasts. Journal of Translational Medicine: 1-11, Doi: 10.1186/s12967-015-0603-0.

48. Cordes, K.R., Sheehy, N.T., White, M.P., Berry, E.C., Morton, S.U., Muth, A.N., et al., 2009. miR-145 and miR-143 regulate smooth muscle cell fate and plasticity. Nature 460(August), Doi: 10.1038/nature08195.

49. Xihua, L.I.N., Shengjie, T., Weiwei, G.U.I., Matro, E., Tingting, T.A.O., Lin, L.I., et al., 2018. Circulating miR-143-3p inhibition protects against insulin resistance in Metabolic Syndrome via targeting of the insulin-like growth factor 2 receptor. Translational Research 205: 33-43, Doi: 10.1016/j.trsl.2018.09.006.

50. Ghosh, P., Dahms, N.M., Kornfeld, S., 2003. Mannose 6-phosphate receptors: New twists in the tale. Nature Reviews Molecular Cell Biology 4(3): 202-12, Doi: 10.1038/nrm1050.

51. Zaina, S., Nilsson, J., 2003. Insulin-like growth factor II and its receptors in atherosclerosis and in conditions predisposing to atherosclerosis. Current Opinion in Lipidology 14(5): 483-9, Doi: 10.1097/00041433-200310000-00009.

52. Beneit, N., Luis, J., Ventura, M., Longás, C.R., Escribano, Ó., Gómez, G.G., et al., 2018. Potential role of insulin receptor isoforms and IGF receptors in plaque instability of human and experimental atherosclerosis. Cardiovascular Diabetology: 1-14, Doi: 10.1186/s12933-018-0675-2.

53. Huang, C., Kuo, W., Yeh, Y., Ho, T., Lin, J., Lin, D., et al., 2014. ANG II promotes IGF-IIR expression and cardiomyocyte apoptosis by inhibiting HSF1 via JNK activation and SIRT1 degradation: 1262-74, Doi: $10.1038 /$ cdd.2014.46.

\section{Figures}

\section{Figure 1}

\section{miR-155-5p and miR-143-3p expression in the aorta of the mouse model of atherosclerosis. (A)}

Representative images of the Oil Red 0 staining from the aortic roots of the 6 groups of experimental mouse model of atherosclerosis. (B) Graphics of quantification of the percentage of lipid accumulation, lesion area and stenosis of aortic root from the mouse model. Magnification 40X (scale bar $=200 \mu \mathrm{m}$ );

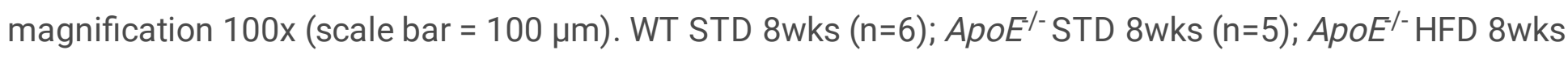

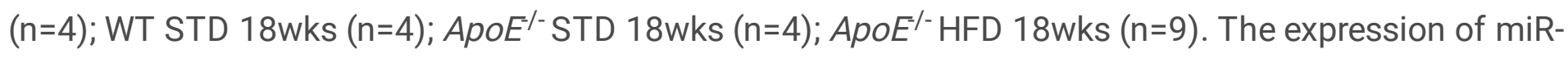
$155-5 p(C)$ and miR-143-3p (D) in the aorta of the three experimental groups at 8 (right graphic) and 18 wks (left graphic) of STD or HFD was analysed by qPCR. RQ $=2^{-D D C t}$; WT= Wild type group; STD= standard type diet; $A p o E^{--}=A p o E$ deficient mice; $\mathrm{HFD}=$ high-fat diet; wks= weeks. qPCR miR-155-5p: WT

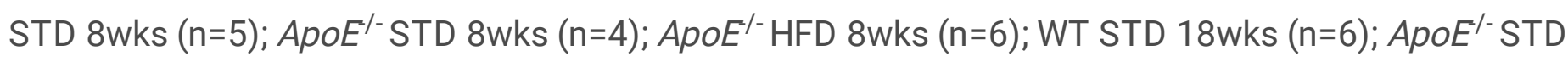


18wks (n=6); ApoE ${ }^{-1}$ HFD 18wks (n=7). qPCR miR-143-3p: WT STD 8wks ( $\left.\mathrm{n}=7\right) ; A p o E^{\prime-}$ STD 8wks $(\mathrm{n}=5)$; $A p o E^{/-}$HFD 8wks (n=8); WT STD 18wks (n=7); ApoE $E^{\prime-}$ STD 18wks (n=8); ApoE $E^{--}$HFD 18wks (n=8).

\section{Figure 2}

Characterization of the atherosclerotic plaques and miRNA expression of the human vascular samples.

(A) This figure shows representative images of the Masson trichomic staining from the aorta of healthy subjects and patients with fibrolipidic lesions and the carotid artery from ACA patients. Magnification $100 x$ (scale bar $=100 \mu \mathrm{m}$ ). Graphics corresponding to the levels of the miR-155-5p $(B)$ and miR-143-3p (C) by qPCR. FL= fibrolipidic plaque; $A C A=$ advanced carotid atherosclerotic plaque; $\mathrm{M}=$ media; $\mathrm{F}=$ fibrous; $S=$ shoulder; $A$ = atheroma. qPCR miR-155-5p: Healthy subjects $(n=3)$; FL patients $(n=5) ; A C A$ patients $(n=11)$. qPCR miR-143-3p: Healthy subjects $(n=5)$; fibrolipidic patients $(n=6)$; ACA patients $(n=14)$. 


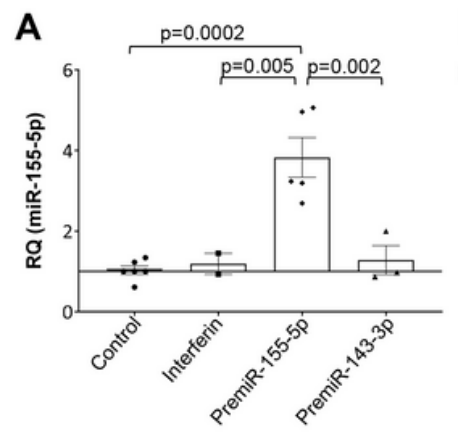

D
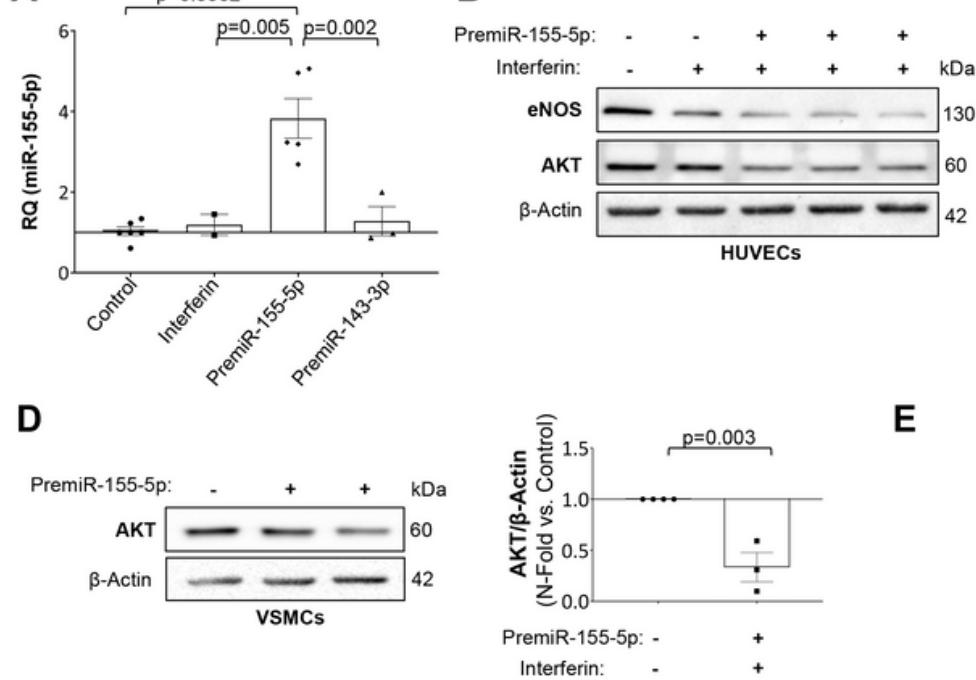

Interferin: -
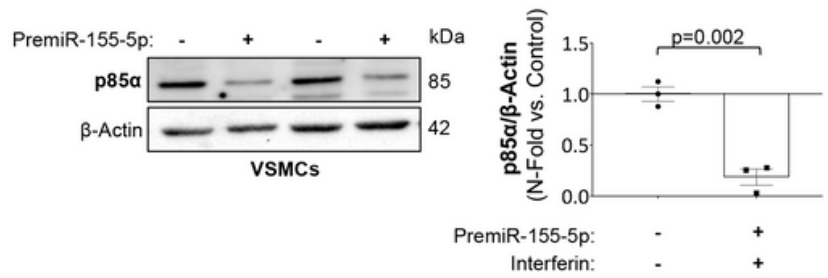

Figure 3
C

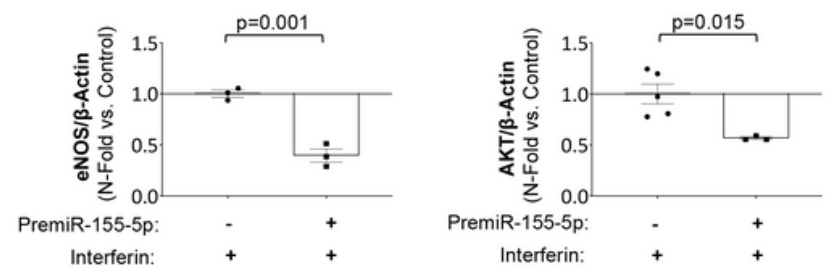

E
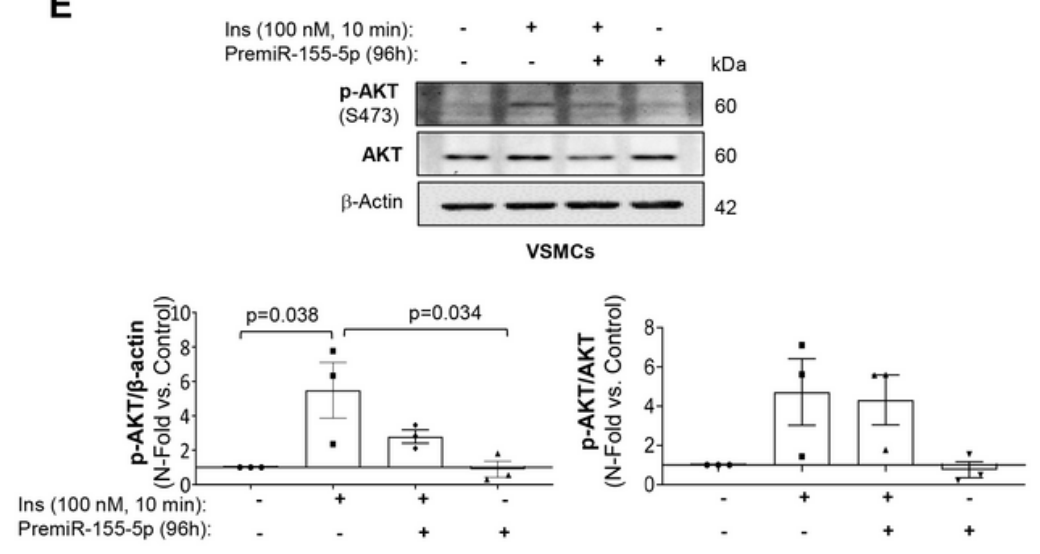

\section{Figure 3}

miR-155-5p reduced AKT expression and its activation in vascular cells. (A) HUVEC and VSMC cell lines were transfected with miR-155-5p mimic for 72h. The increase in miRNA expression was measured by qPCR in HUVECs. (B and $\mathbf{C}$ ) The silencing effect the miRNA has on its targets AKT and eNOS was analysed by Western blot in HUVECs $96 \mathrm{~h}$ after transfection. (D) p85a and AKT was analysed by Western blot in VSMCs $96 \mathrm{~h}$ after transfection. (E) To confirm that miR-155-5p prevents AKT activation, VSMCs were transfected for $96 \mathrm{~h}$ and deprived for $6 \mathrm{~h}$. After that time, the cells were treated with $100 \mathrm{nM}$ insulin for 10 minutes and p-AKT (S473), AKT and $\beta$-actin levels were measured by Western blot. HUVECs = human umbilical vein endothelial cells; VSMCs = vascular smooth muscle cells; eNOS = endothelial nitric oxide synthase; $A K T=$ protein kinase $B ; p 85 a=$ phosphoinositide-3-kinase regulatory subunit 1 ; Ins= insulin. All the in vitro experiments were performed in triplicate. 
AKT levels are downregulated in the human samples and the mouse model where its activity is also impaired. (A) The levels of AKT were measured by Western blot in the aorta artery of the 18 weeks-fed mice groups. (B) Representative images of AKT levels measured by immunohistochemistry in the aorta and carotid human samples. Magnification 100x (scale bar $=100 \mu \mathrm{m}$ ); magnification 200X (scale bar $=$ $50 \mu \mathrm{m}$ ). (C) The correlation between miR-155-5p and AKT expression was set by a Spearman's correlation. (D) And in vivo insulin signalling study was carried out in the mouse model to analyse AKT phosphorylation induced by insulin. The levels of p-AKT (S473), AKT and $\beta$-actin were measured by Western blot of aorta artery samples. WT= Wild type group; STD = standard type diet; $A p o E^{\prime-}=A p o E$ deficient mice; $\mathrm{HFD}=$ high fat diet; $\mathrm{AKT}=$ protein kinase $\mathrm{B} ; \mathrm{FL}=$ fibrolipidic plaque; $\mathrm{ACA}=$ advanced carotid atherosclerotic plaque. Mouse model AKT Western blot: WT STD 18wks $(n=3) ; A p o E^{-1-}$ STD 18wks $(n=3)$; ApoE $E^{/-}$HFD 18wks ( $\left.n=3\right)$; AKT immunohistochemistry: Healthy subjects $(n=5)$; FL patients $(n=4)$; ACA $(n=12)$. miR-155-5p-AKT correlation $(n=14)$. In vivo signalling experiment: WT STD 18wks saline $(n=4)$;

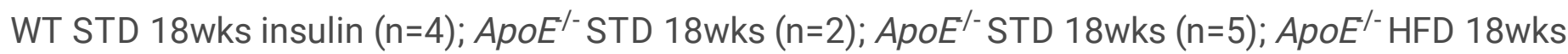
$(\mathrm{n}=3) ; A p o E^{/-}$HFD $18 \mathrm{wks}(\mathrm{n}=3)$.

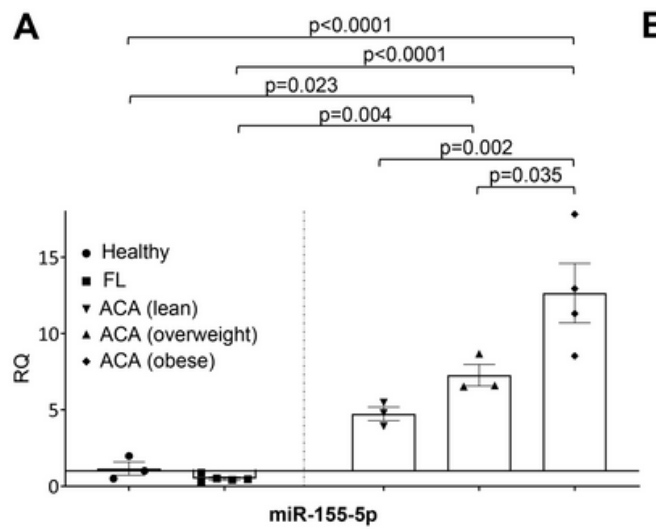

c
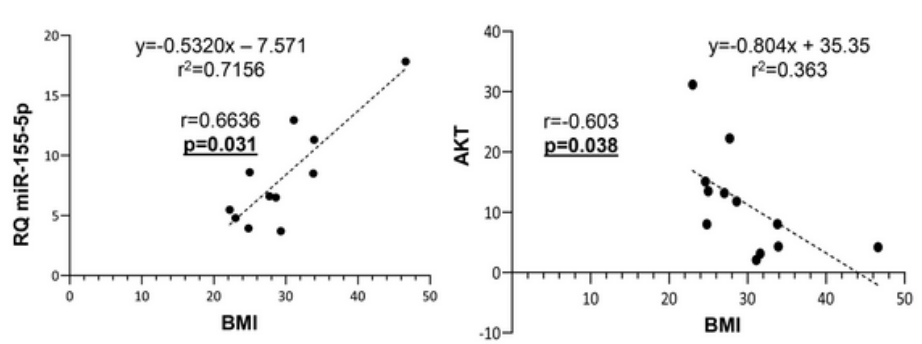

B
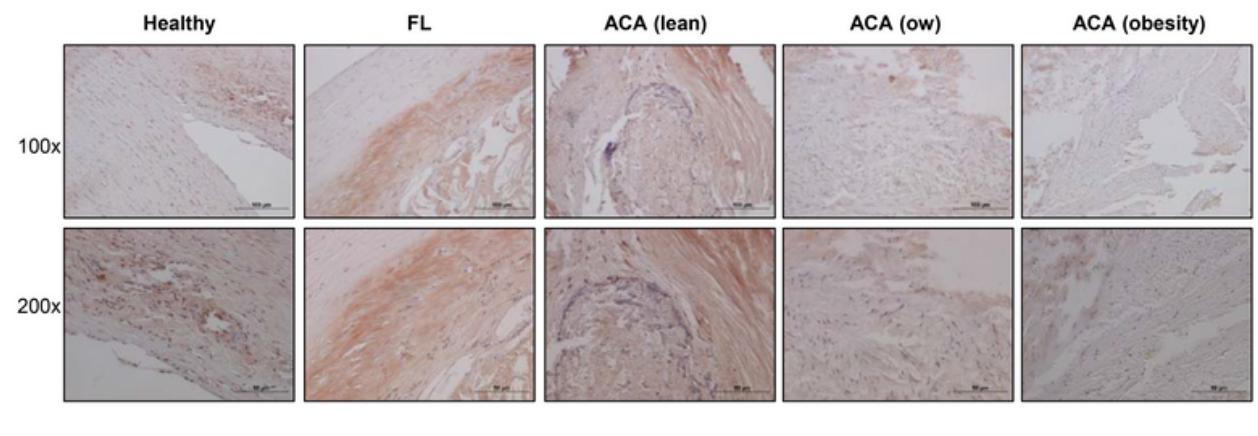

AKT IHC

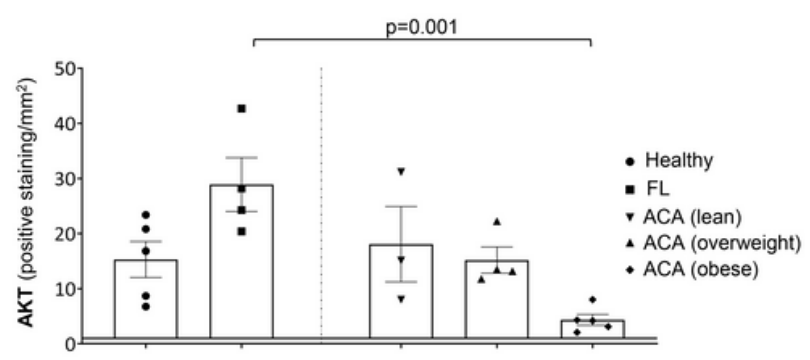

Figure 5 
Figure 5

miR-155-5p and AKT expression correlated with the body mass index in human samples. ACA patients were separated by their BMI following the criteria established by the World Health Organization. (A) miR155-5p expression was quantified by qPCR and (B) AKT expression was measured by immunohistochemistry. Magnification 100x (scale bar $=100 \mu \mathrm{m}$ ); magnification 200X (scale bar $=50$ $\mu \mathrm{m}$ ). The correlation between (C) miR-155-5p and BMI (left graph), and AKT and BMI (right graph) was tested by a Spearman's correlation. $A K T$ = protein kinase $B ; F L=$ fibrolipidic plaque; $A C A=$ advanced carotid atherosclerotic plaque; ow = overweight; $\mathrm{IHC}=$ immunohistochemistry; $\mathrm{BMI}=$ body mass index. miR-155-5p qPCR: Healthy ( $n=3)$; FL ( $n=5)$; ACA (lean) ( $n=3)$; ACA (overweight) $(n=3) ;$ ACA (obese) $(n=4)$. AKT immunohistochemistry: Healthy $(n=5) ; F L(n=4) ; A C A$ (lean) $(n=3) ; A C A$ (overweight) $(n=4) ;$ ACA (obese) $(n=5)$. Correlation between miR-155-5p and BMI $(n=11)$ and between AKT and BMI $(n=12)$.

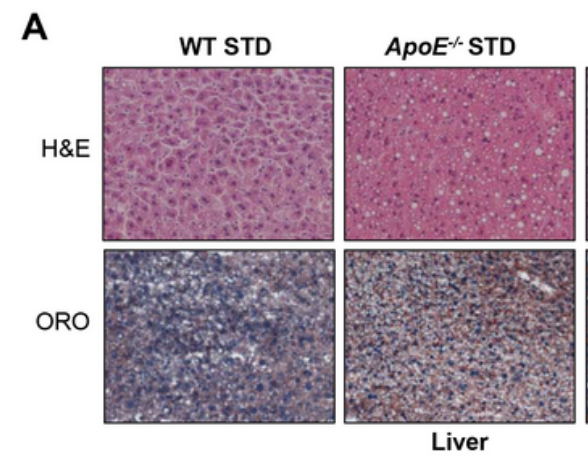

B

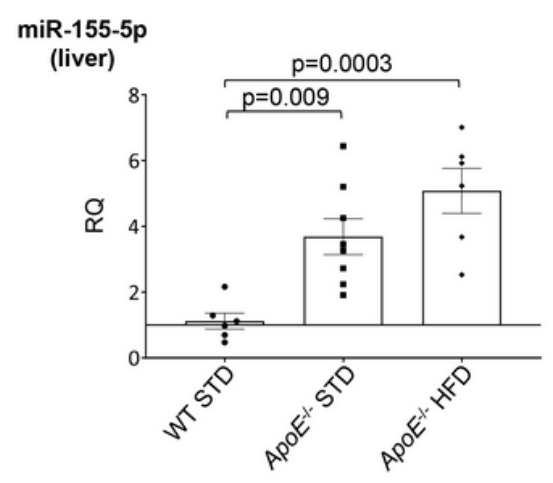

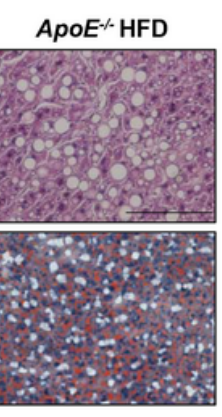

C

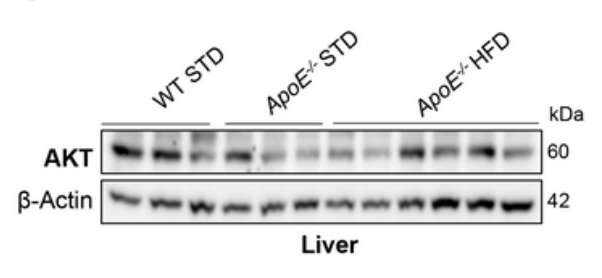

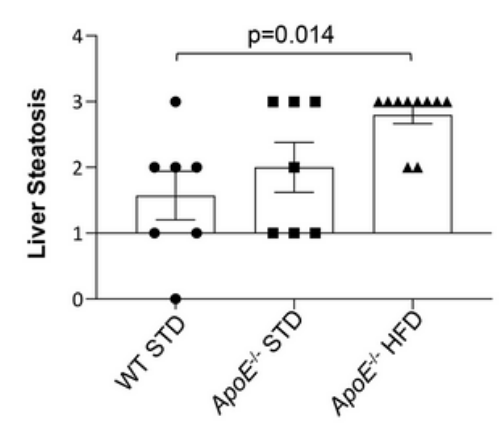

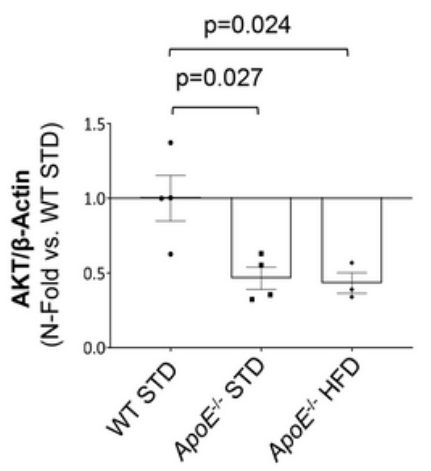

Figure 6

\section{Figure 6}


miR-155-5p is overexpressed and AKT downregulated in the liver of the mouse model after 18 weeks of high-fat diet. (A) Representative images of H\&E staining to perform liver histological analysis (upper) and of Oil Red O staining to evaluate hepatic-specific lipid content. Magnification 200X (scale bar $=25 \mu \mathrm{m}$ ). At right, its corresponding graph that show the quantification of liver steatosis. The levels of circulating miR-155-5p (B) were measured in in the liver of the mice by qPCR and AKT protein levels (C) in liver were measured by Western blot. WT= Wild type group; $S T D=$ standard type diet; $A p o E^{\prime-}=A p o E$ deficient mice; $\mathrm{HFD}=$ high-fat diet; $A K T=$ protein kinase $B$. Image and its quatification steatosis liver: WT STD 18 wks $(\mathrm{n}=7), \mathrm{ApoE}^{-/-}$STD $18 \mathrm{wks}(\mathrm{n}=7)$ and $\operatorname{ApoE}^{/-} \mathrm{HFD}(\mathrm{n}=10)$ mice after 18 weeks on the diet. miR-155-5p qPCR liver: WT STD 18wks (n=6); $A p o E^{--}$STD 18wks (n=8); ApoE ${ }^{--}$HFD 18wks (n=6). Western blot of AKT

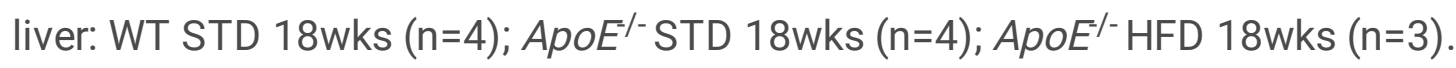

\section{Figure 7}

miR-143-3p protects against apoptosis in endothelial and vascular smooth muscle cells by targeting IGFIIR. To confirm the role miR-143-3p in atherosclerosis progression, we performed in vitro experiments in HUVECs and VSMCs. (A) First, miR-143-3p was overexpressed using a miRNA mimic in HUVECs (left graph) and VSMCs (right graph) after a 72h transfection. (B) Effect of miR-143-3p on IGF-IIR was quantified by Western blot after a $96 \mathrm{~h}$ transfection with the mimic in HUVECs and VSMCs. IGF-IIR levels were measured in the mouse model by Western blot (C) and in human samples by immunohistochemistry (D). (E) In order to confirm miR-143-3p as a protector against apoptosis HUVECs and VSMCs cell lines were treated with a proapoptotic signal after a 96h transfection with the mimic. HUVECs were deprived from FBS for $6 \mathrm{~h}$ with or without miRNA overexpression (left graph). VSMCs were pre-treated with a $2 \mathrm{~h}$ FBS-deprivation and then treated with $100 \mathrm{nM}$ thapsigargin for $2 \mathrm{~h}$ with or without miRNA overexpression (right graph). Apoptosis was measured by the expression of cleaved caspase 3 by Western blot in both cell lines. HUVECs= human umbilical vein endothelial cells; VSMCs= vascular smooth muscle cells; IGF$\mathrm{IIR}=$ insulin-like growth factor type 2 receptor; $\mathrm{WT}=$ Wild type group; $\mathrm{STD}=$ standard type diet; $A p o E^{-{ }^{-}=}=$ $A p o E$ deficient mice; $\mathrm{HFD}=$ high fat diet; $\mathrm{IHC}=$ immunohistochemistry; $\mathrm{M}=$ =media; $\mathrm{F}=$ fibrous; $\mathrm{S}=S$ Shoulder; $\mathrm{FBS}=$ foetal bovine serum. All the in vitro experiments have been performed at least 3 times $(n=3)$. Western blot of IGF-IIR: WT STD 18wks (n=3); ApoE ${ }^{--}$STD 18wks (n=3); ApoE ${ }^{\prime-}$ HFD 18wks (n=3). Immunohistochemistry of IGF-IIR: Media ( $n=44)$; Fibrous $(n=49)$; Shoulder $(n=19)$.

\section{Figure 8}


Graphical scheme of the role of miR-155-5p and miR-143-3p in advanced atherosclerosis. The overexpression of miR-155-5p and consequently, the downregulation of its target AKT in resident vascular cells of advanced atherosclerotic plaques produces vascular insulin resistance. Furthermore, in advanced atherosclerosis in humans and in mice we have found miR-143-3p downregulation and an overexpression of its target, IGF-IIR, which could favour the apoptosis of VSMCs present in advanced lesions and lead their rupture.

\section{Supplementary Files}

This is a list of supplementary files associated with this preprint. Click to download.

- SupplementalData.GonzlezLpezetal.CardiovascDiabetol22.pdf 